\title{
Nephroblastoma with Atypical Pelvic Metastasis About A Case
}

\author{
B. Bannar ${ }^{*}$, M. Badraoui, I. Zouita, D. Basraoui, H. Jalal
}

Radiology Department, Mother and Child Hospital CHU Mohamed VI Marrakech Morocco

DOI: $10.36347 /$ sjmcr.2020.v08i03.019

| Received: 29.02.2020 | Accepted: 07.03.2020 | Published: 16.03.2020

*Corresponding author: B. Bannar

\section{Abstract}

The nephroblastoma is the most common malignant tumor in children, but the metastatic localization is rare, We report in this letter the case of a girl 6 year old. Who benefetied Abdominal and pelvic ultrasound showed a retro-peritoneal mass associated with another pelvic mass requiring a CT scan, which objectified a right retro-peritoneal mass with capsular intrusion, adrenal infiltration and of the liver on the ipsilateral side evoking a nephroblastoma associated with a second centro-pelvic mass that may be related to a secondary,location. Our patient underwent a biopsy which confirmed the diagnosis of nephroblastoma and its secondary location.

Keywords: Nephroblastoma, metastasis, ovary

Copyright @ 2020: This is an open-access article distributed under the terms of the Creative Commons Attribution license which permits unrestricted use, distribution, and reproduction in any medium for non-commercial use (NonCommercial, or CC-BY-NC) provided the original author and source are credited.

\section{INTRODUCTION}

The nephroblastoma is the most common malignant tumor in children, but the metastatic localization is rare, it is still found in the child of less than 6 years. The diagnosis is mainly based on +++ imaging (first-line ultrasound). The biopsy is contraindicated and the treatment is surgical. Our goal is to present an unusual site of secondary localization of nephroblastoma in children.

\section{Presentation of Case}

We report in this letter the case of a girl 6 year old. Who has abdominal pain without other associated signs. The clinical examination showed: a large mass of the right hypochondrium, extended to the flank and to the lumbar fossa: evolving for 2 months without associated signs. The biological evaluation was made without any abnormalities, except normochromic anemia.

Abdominal and pelvic ultrasound (figure 1 and 2) showed a retro-peritoneal mass that could be related to a nephroblastoma or neuroblastoma associated with another pelvic mass requiring a CT scan, which objectified a right retro-peritoneal mass with capsular intrusion, adrenal infiltration and of the liver on the ipsilateral side evoking a nephroblastoma associated with a second centro-pelvic mass that may be related to a secondary location.

Ultrasound can show the tumor mass in the pelvic region, but topographic diagnosis in the ovaries is difficult if the volume of the mass is increased.

The realization of a pelvic abdominal MRI is useful in order to be able to specify the ovarian origin of the tumor mass. 


\section{RESULTS}

1/Abdominal and pelvic echography

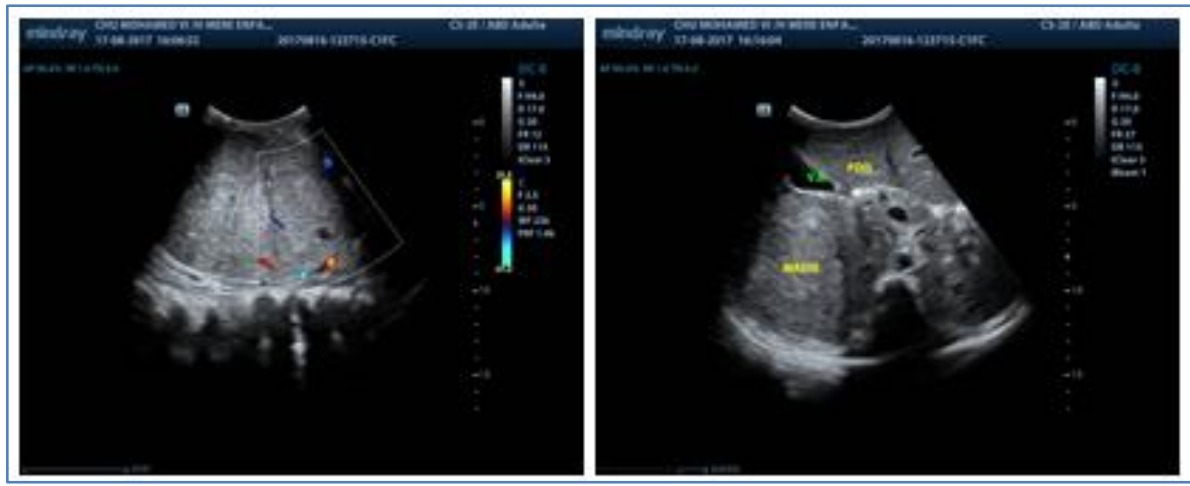

Fig-1: Bulky tissue mass occupying the right renal lodge. The right kidney could not be visualized

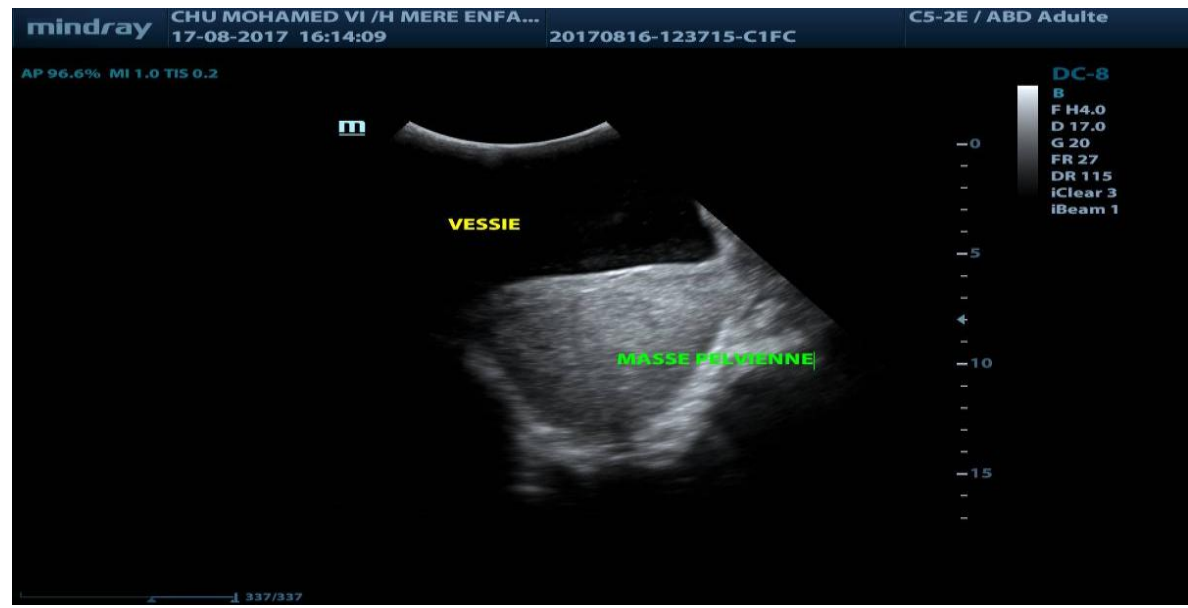

Fig-2: Presence of a second retro-vesical mass with the same characteristics

2/Abdominal and pelvic CT scan with contrast product: axial, coronal and sagittal sections

Voluminous mass in the right kidney enhanced after contrast injection, associated with a second bilateral ovarian metastatic localization (figure 3) Adrenals are normal.

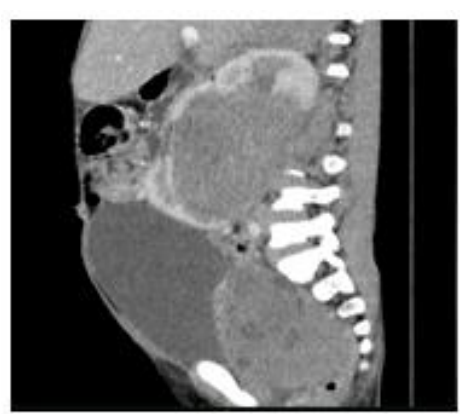

\section{Therapeutic management}

6 cures of chemotherapy for the reduction of the tumor mass according to the CFA protocol

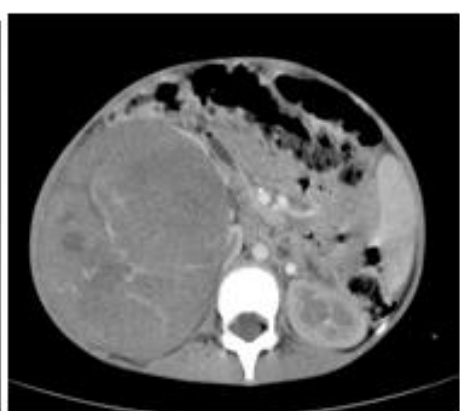

Fig-3

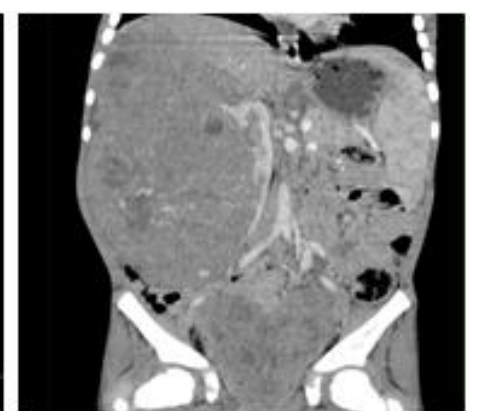

\section{Ultrasound control}

Total disappearance of the two ovarian masses with paucifolic ovaries of size limit (figure 4) measuring: Right $1.53 \times 0.84 \times 5.3 \mathrm{~cm}$, Left $1.15 \times 0.82$ $\mathrm{x} 5 \mathrm{~cm}$ 


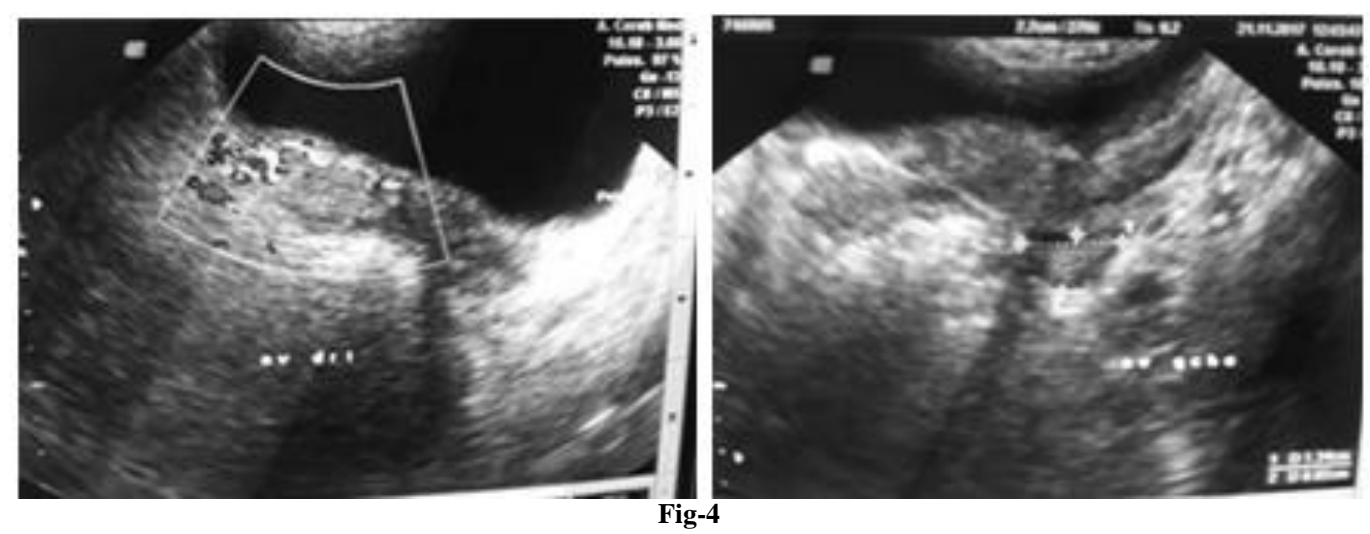

\section{Abdominal and pelvic et scan contol after chemotherapy}

- Decreased size of the renal mass with persistence of an upper right polar tumor residue.

- Disappearance of the ovarian masses (figure5).

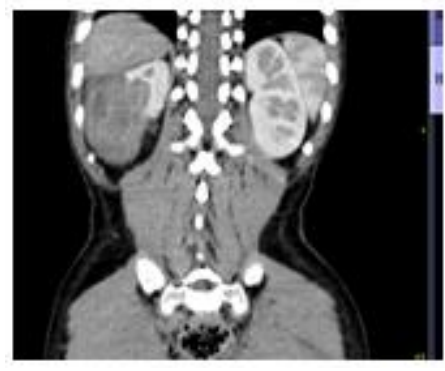

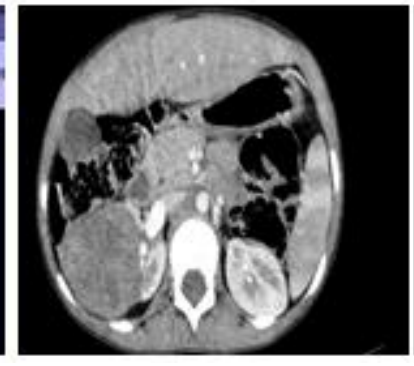

Fig-5

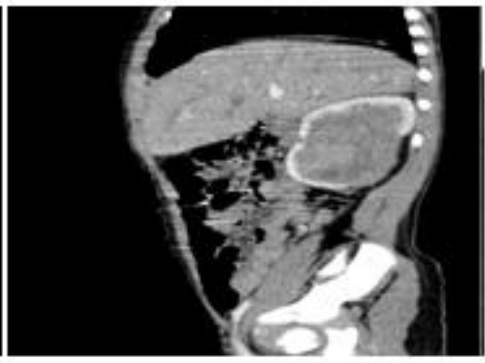

The realization of a pelvic abdominal CT or

Follow-up of the care Total right ureteronephrectomy was done at the service of pediatric surgery

\section{DISCUSSION}

The ovaries are a frequent site of metastases. About 5 to $15 \%$ of malignant ovarian lesions are metastases. The majority of primitive sites are of gastrointestinal origin, notably the stomach and the colon. The biliary origin is rarely described in the literature. The occurrence of these metastases at a distance from the discovery of the original tumor poses a real problem of differential diagnosis with a primary ovarian tumor. The authors report 2 cases of ovarian metastases of biliary origin.

Two similar cases of ovarian metastases of nephroblastoma was described and diagnosed by the team of Professor Rachida Dafiri (Pediatric Radio Service, children's hospital, IBN SINA CHU, RABAT): 2 children 9 years and 7 years.

\section{Conclusion}

Ultrasound can show the tumor mass in the pelvic region, but topographic diagnosis in the ovaries is difficult if the volume of the mass is increased.

\section{REFERENCES}

1. Ovarian metastases of biliary origin: 2 cases and review of the literature. Pan Afr Med J. 2013; 16 : 44

2. Les métastases ovariennes d'origine biliaire: 2 cas avec revue de la literature(Ovarian metastases of biliary origin: 2 cases and review of the literature) Imane Kamaoui,1,\& Mustapha Maaroufi,1 Benjalloun el Bachir, 2 Hanane Ouzaa,1 et Siham Tizniti1 Author information Article notes Copyright and License information Disclaimer

3. Jarvi K, Kelty CJ, Thomas WEG, Gillespie A. Bilateral ovarian metastases from carcinoma of the gallbladder. Gynecologic Oncology. 2006;103(1):361.I

4. Sharma P, Jaffe PE, Bhattacharyya A. Metastatic cholangiocarcinoma presenting as ovarian cancer: a rare Krukenberg tumour. Am J Gastroenterol. 1997;92(3):531. 